\title{
Trade-off between phase-noise and signal quadrature in unilaterally coupled
} oscillators

\author{
Djurhuus, Torsten; Krozer, Viktor; Vidkjær, Jens; Johansen, Tom Keinicke
}

Published in:

Microwave Symposium Digest, 2005 IEEE MTT-S International

Link to article, DOI:

10.1109/MWSYM.2005.1516761

Publication date:

2005

Document Version

Publisher's PDF, also known as Version of record

Link back to DTU Orbit

Citation $(A P A)$ :

Djurhuus, T., Krozer, V., Vidkjær, J., \& Johansen, T. K. (2005). Trade-off between phase-noise and signal quadrature in unilaterally coupled oscillators. In Microwave Symposium Digest, 2005 IEEE MTT-S International (pp. 883-886). IEEE. https://doi.org/10.1109/MWSYM.2005.1516761

\section{General rights}

Copyright and moral rights for the publications made accessible in the public portal are retained by the authors and/or other copyright owners and it is a condition of accessing publications that users recognise and abide by the legal requirements associated with these rights.

- Users may download and print one copy of any publication from the public portal for the purpose of private study or research.

- You may not further distribute the material or use it for any profit-making activity or commercial gain

- You may freely distribute the URL identifying the publication in the public portal 


\title{
Trade-Off Between Phase-Noise and Signal Quadrature in Unilaterally Coupled Oscillators
}

\author{
Torsten Djurhuus, Viktor Krozer, Jens Vidkjær and Tom K. Johansen \\ Technical University of Denmark,Oersted•DTU Department, Electromagnetic Systems, \\ Oersteds Plads 348, 2800 Kgs. Lyngby, Denmark, Phone:+45-45253770, (e-mail:tdj@oersted.dtu.dk)
}

\begin{abstract}
We present a comprehensive nonlinear analysis of coupled oscillators and examine the trade-off between phase-noise of the oscillator and the quadrature precision. We show that asymmetry gives rise to amplitude and phase imbalance which are proportional to the inverse and inverse square, respectively, of the relative coupling strength. It is shown that the level of AM-PM is determined by the nonlinearity of the coupling transconductance. The $3 \mathrm{~dB}$ noise reduction in close-to-carrier phase-noise in quadrature oscillators due to mutual coupling is lost to the extra AM-PM noise for large coupling strengths. The additional contribution of the internal noise sources in the coupling circuit together with the AM-PM noise contribution explains why the 3dB noise reduction is rarely seen in measurements of this particular circuit.
\end{abstract}

Index Terms-Oscillator, Nonlinear Analysis, Phase-Noise

\section{INTRODUCTION}

Coupled oscillator arrays are increasingly important in microwave and millimeter-wave applications, such as e.g. phased-array systems or quadrature oscillators. The generation of quadrature signals is an essential part of modern RF- and microwave communication receiver architectures such as the zero-IF and image reject receivers. Possible circuit structures producing such signals include a frequency division scheme, poly-phase filters, and oscillators coupled in a ring structure [1]. The first two options require either oscillators running at double the desired carrier frequency, or excellent matching of the filter components. Coupled LC oscillator structures have become the preferred choice for the generation of quadrature signals, as they dispose of the above mentioned difficulties and are in principle capable of generating multi-phase signals. The basic theory of bilaterally coupled oscillators has been presented before. However, the properties of unilaterally coupled oscillators are still not well understood, including the trade-off between phase precision and low phase-noise operation. Also the effects of asymmetry in the individual stages of the oscillators has received only minor attention.

In this paper we shall consider the coupling of two LC differential oscillators in a ring structure with separate bias for the coupling network. This enables to identify the contribution of the coupling stage nonlinearity to the oscillator performance. This circuit, known as a crosscoupled quadrature LC oscillator, is described in detail in
[2]. It delivers two equal amplitude signals that are ideally 90 degrees out of phase, however, any asymmetry present in the circuit will result in a departure from this ideal [3]. Analysis of this circuit has been attempted before [4], [5], [6], [7] but a qualitative analysis describing the impact of the different circuit parameters including asymmetry on signal quadrature and close-to-carrier phase noise is still missing.

It is the aim of this paper to present such an analysis leading to a qualitative explanation of the trade-off between noise performance and the precision of signal quadrature. The analysis presented also identifies the contribution of the nonlinearity in the coupling stages to the AM-PM conversion noise close to the carrier. It is shown that the noise reduction due to mutual coupling of oscillators is counter-balanced by the increase of the noise which is the consequence of the coupling circuit nonlinearity.

\section{ANALYSis of THE DyNAMICS OF UNILATERALLY COUPLED OSCILLATORS}

A typical quadrature oscillator structure is illustrated in Fig. 1 including a block diagram of the oscillator. The top figure illustrates a full transistor implementation of the oscillator, where the coupling transconductance stage has been realized as a generic voltage controlled current source (VCCS). The current voltage characteristic of these VCCS represents the current voltage characteristic of the differential pair. The introduction of VCCS is needed in order to be able to control the nonlinearity of the coupling stages. A nonlinear analysis of the circuit leads to coupled differential equations describing the dynamical behaviour of the oscillator, which can be used for the determination of the phase-noise and of phase precision.

An out-of-phase current is injected into each section by the coupling transconductance. This results in a shift of operating frequency away from the resonator natural frequencies of the individual oscillators. The frequency of an oscillator with a parallel LC tank $\omega_{1}$ can be related to the resonator phase shift $\psi$ through

$$
\frac{\omega_{1}}{\omega_{0}}= \pm \frac{\tan (\psi)}{2 Q}+\frac{1}{2 Q} \sqrt{4 Q^{2}+\tan ^{2}(\psi)}
$$

where only the positive frequencies are considered, $Q$ is the resonator loaded quality factor and $\omega_{0}$ is the resonator 


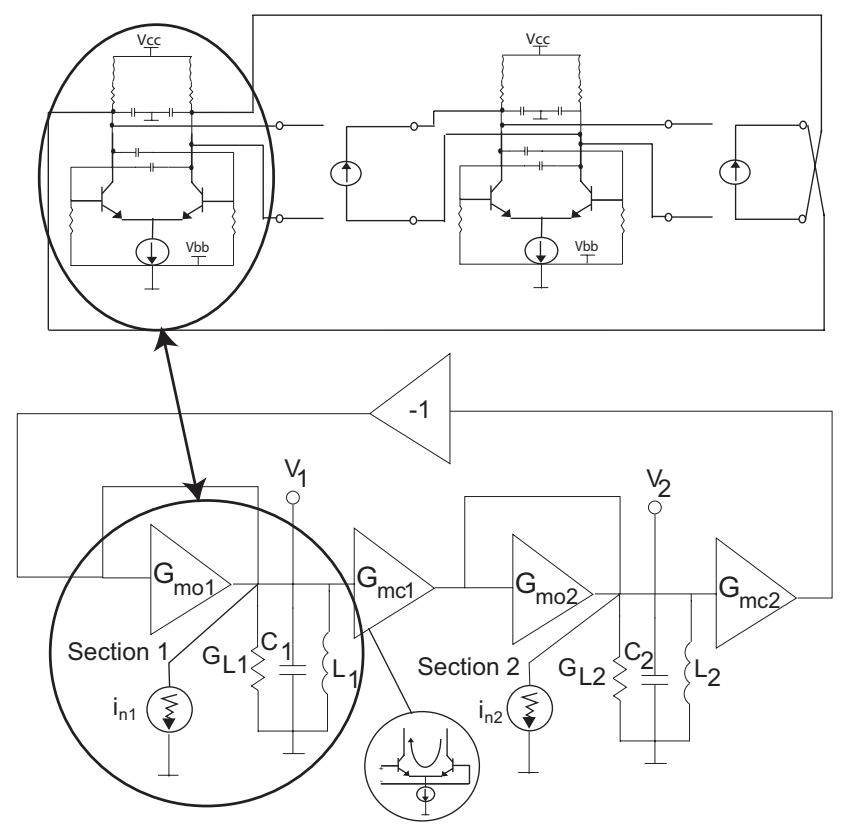

Fig. 1. Cross-coupled quadrature harmonic oscillator schematic and single ended block diagram. As indicated, the gain blocks are usually implemented by a transistor differential pair. $G_{m o}$ and $G_{m c}$ are the oscillator negative conductance and coupling transconductance, respectively. The top triangle symbolize the $180^{\circ}$ phase shift of the cross-coupling. Currents $i_{n 1}$ and $i_{n 2}$ represent resonator thermal noise.

natural frequency. The complex resonator admittance phasor $Y_{R}(j \omega)$ can be linearized around $\omega_{1}$ yielding

$$
\begin{aligned}
Y_{R}(\omega) & =G_{L}+j G_{L} \tan (\psi) \\
& +j G_{L} \sqrt{4 Q^{2}+\tan ^{2}\left(\psi_{i}\right)} \frac{\left(\omega-\omega_{1}\right)}{\omega_{1}}
\end{aligned}
$$

where $\omega$ is the instantaneous operating frequency. Node voltages in Fig. 1 have the form $V_{1}=\Re\left\{A_{1} e^{j\left(\omega_{1} t+\phi_{1}\right)}\right\}$ and $V_{2}=\Re\left\{j A_{2} e^{j\left(\omega_{1} t+\phi_{2}\right)}\right\}$, where the amplitude and phase modulations are slowly varying (quasi-static) functions of time. The set of equations describing the amplitude and phase dynamics of the circuit in Fig. 1 is derived considering a third order nonlinearity for both the individual oscillators (van der Pol oscillator) and the coupling amplifiers leading to a set of averaged coupled differential equations. Using Kurokawa's substitution together with the expression in (2) the following system of equations is established (see [4] and [6] for details of the derivation)

$$
\begin{aligned}
& \frac{d A_{i}}{d \tau}=\mu_{i}\left[1-\left(\frac{A_{i}}{\alpha_{i}}\right)^{2}\right] A_{i}-\frac{G_{m c, j}}{G_{L i}} \sin \left(\phi_{j}-\phi_{i}\right) A_{j} \\
& \frac{d \phi_{i}}{d \tau}=\frac{2 Q_{i}}{\omega_{0 i}} \Delta \omega_{i}+\frac{G_{m c, j}}{G_{L i}} \cos \left(\phi_{j}-\phi_{i}\right) \frac{A_{j}}{A_{i}} \\
& \quad(i, j) \in\{1,2\}, \quad i \neq j
\end{aligned}
$$

where the subscripts $i, j$ refer to the different oscillator sections. The first harmonic large-signal transconductance of the coupling stage is $G_{m c}=g_{m c}\left(1-0.75 K_{c} A^{2}\right)$, with $K_{c}$ a constant and $g_{m c}$ the small-signal transconductance of the coupling stages, $\mu=\left(g_{m d}-G_{L}\right) / G_{L}$, where $g_{m d}$ stands for the small-signal transconductance of the oscillator circuit, $\alpha$ is the free running amplitude, and $\Delta \omega=\omega_{1}-\omega_{0}$. We have also used the time normalization $\tau=t \times \omega_{3 d B}$ with $\omega_{3 d B} \approx \omega_{1} /(2 Q)$. The assumption that the amplitude and phase transients can be considered constant within one oscillation period, implies a resonator quality factor much greater than one [4].

Equations (3) and (4) contain contributions of the all nonlinearities and can be used for the determination of the noise performance of the oscillator. The above equations are now used to analyze the oscillator performance in the next section.

\section{Phase-Noise and Phase Precision Analysis OF UNILATERALly COUPLED OSCILlatoRs \\ A. Impact of Circuit Asymmetry on Amplitude and Phase Imbalance}

Any asymmetry in the coupling circuits or the individual oscillators will result in a steady-state operation of the unilaterally coupled oscillators that differs from the ideal. Here we focus on small deviations in the oscillator resonance frequencies originating from small variations of the tank circuit inductance and/or capacitance. For example, in the case of inductance variations this corresponds to the case where $L_{1}=L_{2}(1+\kappa)$, (see fig. 1), with $|\kappa| \ll$ 1 representing the asymmetry. The steady-state is now determined by setting the left-hand side in (3) and (4) equal to zero, which after some algebraic manipulations results in the following equations for the steady-state performance (hats denote steady-state variables)

$$
\begin{aligned}
\frac{\hat{A}_{2}}{\hat{A}_{1}} & =\sqrt{\frac{\tan \left(\psi_{1}\right)}{\tan \left(\psi_{2}\right)} \frac{\widehat{G}_{m c 1}}{\widehat{G}_{m c 2}}} \\
\sin (\Delta \hat{\phi}) & =\frac{\frac{\hat{A}_{1}}{\hat{A}_{2}}-\frac{\hat{A}_{2}}{\hat{A}_{1}}}{2 \frac{\zeta}{\mu}}
\end{aligned}
$$

with $\zeta=\widehat{G}_{m c} / G_{L}=\tan (\psi)$ denoting the coupling strength and $\Delta \hat{\phi}=\hat{\phi}_{1}-\hat{\phi}_{2}$. The expressions in equations (5) and (6) can be simplified, assuming very small deviations from nominal and using (1) to relate the frequency and resonator phase shift,

$$
\begin{aligned}
\frac{\hat{A}_{2}}{\hat{A}_{1}} & =1+\epsilon \approx \frac{Q \kappa}{2 \zeta} \cdot \frac{\mu}{1-\mu} \\
\sin (\Delta \hat{\phi}) & \approx \frac{Q \kappa}{2 \zeta^{2}} \cdot \frac{\mu}{1-\mu}
\end{aligned}
$$

Equations (7) and (8) determine the amplitude and phase deviations of the individual stages as a function of the inductor asymmetry in the circuit. For a relative inductor asymmetry of $\kappa=0.05 \%$ the results from equations (7) 
and (8) are plotted in Fig. 2 and are verified with results from SPECTRE PSS simulation for the case where the individual oscillators in Fig. 1 have been implemented as van der Pol type oscillators.
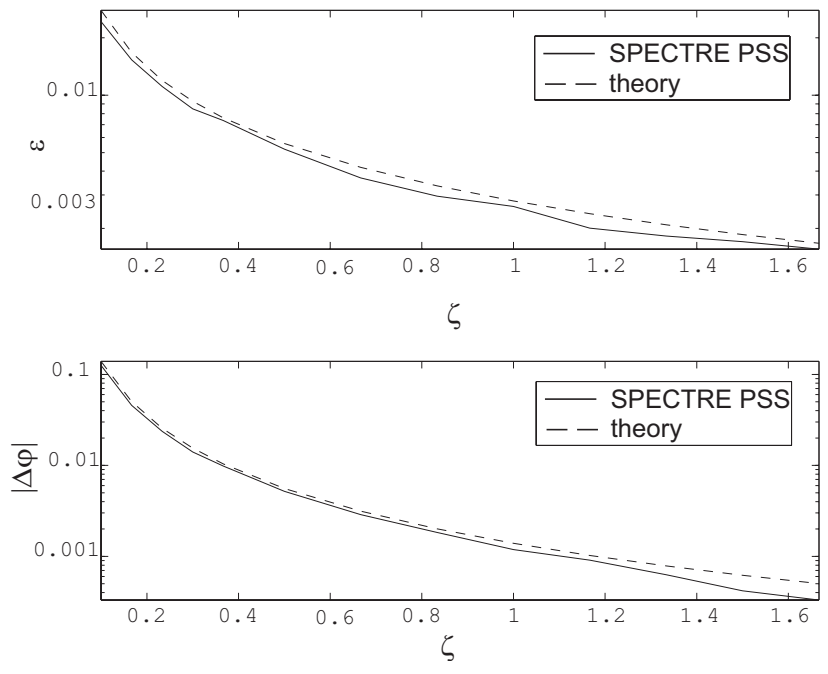

Fig. 2. Amplitude and phase error as a result of a small inductor mismatch $\kappa=0.05 \%$. Comparison between SPECTRE PSS (solid line) simulations and results based on (7) and (8) (dashed line).

Fig. 2 demonstrates that strong coupling is preferable in order to achieve minimum amplitude and phase deviations. From the figure we clearly spot the inverse square law dependence, $1 / \zeta^{2}$, of the phase error on the relative coupling strength, which was first found in [6], where the authors used a graphical phasor analysis to arrive at a similar result. Our work determines additionally the relationship for the amplitude imbalance, which is inversely proportional to the coupling strength, $1 / \zeta$. Similar results can be obtained for asymmetry in the capacitance of the resonators and in the active circuits [8].

The main conclusion from the point of view of asymmetry is that oscillators should be coupled as hard as possible in order to alleviate any amplitude or phase imbalance in the unilaterally coupled oscillator array. However, it is known that the $3 \mathrm{~dB}$ noise reduction from mutual coupling of two oscillators can only be achieved with loose coupling. Therefore, the noise performance of unilaterally coupled oscillators, as indicated in Fig. 1, has to be investigated as a function of the coupling for optimum performance.

\section{B. Impact of Coupling Circuit Nonlinearity on Phase- Noise}

Amplitude and phase-noise in oscillators is caused by a perturbation of the steady-state by small noise-like signals. The resulting state variables in (7) and (8) can be represented by $A_{i}=\hat{A}_{i}+\delta A_{i}$ and $\phi_{i}=\hat{\phi}_{i}+\delta \phi_{i}$, respectively, where $\delta A_{i}$ and $\delta \phi_{i}$ are the resulting slowlyvarying transient oscillator amplitude and phase of the $i$ 'th section. The close-to-carrier phase noise power spectrum is calculated by Fourier transforming the first-variational [7] of the system of equations (3) and (4). For a symmetric circuit $\left(\hat{G}_{m c 1}=\hat{G}_{m c 2}=\hat{G}_{m c}, Q_{1}=Q_{2}=Q\right.$ etc) with a narrow-band Gaussian thermal noise source as the forcing function the following result is obtained

$$
\begin{aligned}
\lim _{\omega_{m} \rightarrow 0}|\delta \phi|^{2} & =\mathfrak{L}_{C}\left(\omega_{m}\right) \\
& =\frac{1}{2}\left[\frac{\omega_{1}}{\sqrt{4 Q^{2}+\tan ^{2}(\psi)}}\right]^{2} \\
& \times\left[1+\left(\frac{\mu_{c}}{\mu}\right)^{2} \tan ^{2}(\psi)\right] \frac{N_{0}}{P_{0} \omega_{m}^{2}} \\
& =\frac{\omega_{1}^{2}}{2\left(4 Q^{2}+\zeta^{2}\right)} \cdot\left[1+\left(\frac{\mu_{c}}{\mu}\right)^{2} \zeta^{2}\right] \frac{N_{0}}{P_{0} \omega_{m}^{2}}
\end{aligned}
$$

where $\mathfrak{L}_{C}\left(\omega_{m}\right)$ denotes the phase-noise of the coupled oscillators at frequency offset $\omega_{m}, N_{0}$ represents the resonator thermal noise power in a $1 \mathrm{~Hz}$ bandwidth, $P_{0}$ is the power delivered to the oscillator load, $\mu_{c}=$ $\left(g_{m c}-\hat{G}_{m c}\right) / \hat{G}_{m c}$. In eq. (9) only the resonator noise is considered for simplicity but the expression can be augmented to include transistor noise by multiplying the equation with the transistor noise factor $\mathrm{F}$ [9].

The trade-off between phase noise and signal quadrature can be analyzed by comparing eq. (7) and (8) with eq. (9). Assuming asymmetry in either of the coupling networks or between the individual oscillators we see from eq. (8) that as the sections are coupled harder (ie. $\zeta$ is increased), the steady-state phase difference will move closer to the ideal 90 degrees, as indicated already in the previous section. However, from eq. 9 we see that this will lead to more AM-PM noise close to the carrier, as $\zeta=\tan (\psi)$. This noise increase is shown in Fig. 3 together with results from Agilent ADS2003C simulations, for different levels of coupling nonlinearity, as represented by the constant $K_{c}$.

The simulations in Fig. 3 are conducted by subtracting the phase noise in $\mathrm{dB}$ at a fixed offset frequency of the quadrature oscillator from the phase noise at the same frequency offset of a single oscillator and then normalizing for the different operating frequencies and amplitudes.

From Fig. 3 and eq. (9) we note the interesting fact that the nonlinearity of the coupling circuits has a strong impact on the level of excess phase noise produced by the circuit. Already relatively weak nonlinearities, as indicated by $K_{c}>0.35$ in Fig. 3, give rise to a phase-noise contribution in excess of $0 \mathrm{~dB}$ relative to a single oscillator. It can therefore be concluded from the above results that a linear coupling circuit is essential for low phase-noise 


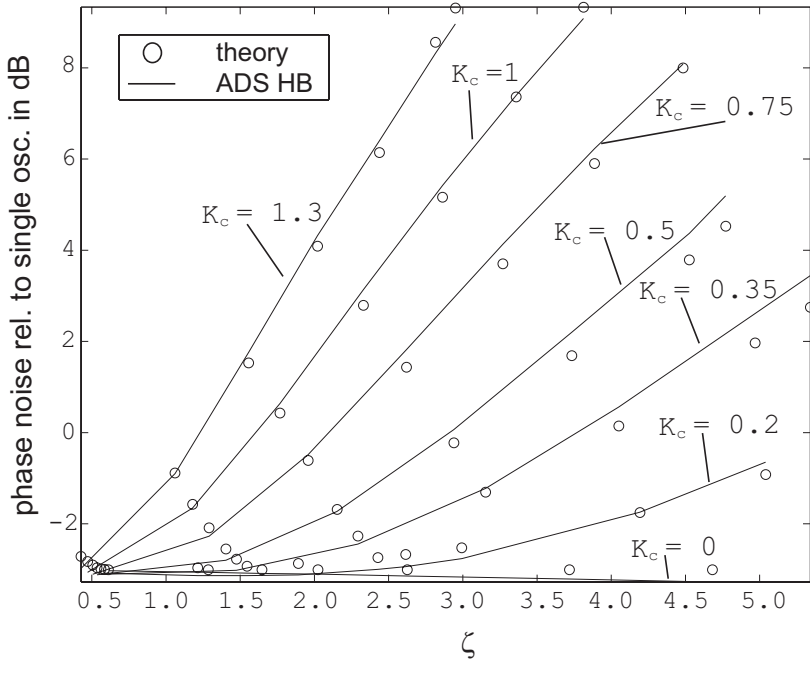

Fig. 3. Agilent ADS2003C phase-noise simulations of a $2 \mathrm{GHz} \mathrm{LC}$ cross-coupled quadrature oscillator in Fig. 1 relative to a single oscillator unit. The coupling transconductance blocks are implemented by ideal third order voltage controlled current sources.

operation. The above results predict that it is possible to completely cancel the close-to-carrier AM-PM noise conversion in unilaterally coupled oscillators using linear coupling stages.

If the constant $K_{c}$, as defined above for $G_{m c}$, is identical for both the oscillator and coupling amplifier transconductances, it can be verified that $\mu_{c} / \mu=1$. For this special case and employing eq. (9) with Leeson's model for phase noise in single oscillators [10] we see that we can define a new Q-factor $Q_{C}$ for the cross-coupled quadrature oscillator as

$$
Q_{C}=\frac{1}{\sqrt{2}} \cos (\psi) \sqrt{4 Q^{2}+\tan ^{2}(\psi)} \approx \underbrace{\sqrt{2}}_{\begin{array}{c}
\text { Mutual } \\
\text { Phase } \\
\text { Locking }
\end{array}} Q \underbrace{\cos (\psi)}_{\begin{array}{c}
\text { AM-PM } \\
\text { Noise } \\
\text { Conversion }
\end{array}} .
$$

It lumps together the effects of mutual phase locking and AM-PM noise conversion. The result in (10) was first found in [7], where the input noise to output phase transfer function was derived through linear analysis. Later, the authors of [6] also derived it using graphical phasor analysis.

In [7], it was found that phase-shifters inserted inbetween the oscillator sections were needed in-order to make noise and quadrature orthogonal entities. This statement still holds. However, the non-linear analysis detailed in this paper reveals a completely new aspect. Inspecting (9) and (10), we see that introducing linear coupling presents yet another way of eliminating the noise/quadrature trade-off. Therefore, we suggest here that the realization of a linear coupling stage leads to full benefit from mutual coupling.

\section{CONCLUSions}

We present a comprehensive nonlinear analysis of unilaterally coupled oscillators and examine the trade-off between phase-noise of the oscillator and the quadrature precision. The analysis has lead to dynamic equations governing the cross-coupled quadrature oscillator in the presence of small noise-like signals. This enables an identification of the previously reported trade-off between phase-noise and signal quadrature precision. Additionally to the previously reported results, we demonstrate here the impact of asymmetry on amplitude imbalance and of the coupling stage nonlinearity on the performance of the unilaterally coupled oscillator. Amplitude imbalance due to asymmetry is inversely proportional to the relative coupling strength, whereas the phase imbalance is proportional as inverse square. Close-to-carrier phase-noise in quadrature oscillators is affected firstly by mutual phase locking leading to a $3 \mathrm{~dB}$ noise reduction and secondly by AM-PM noise conversion, which controls the increase of noise with coupling strength. It is shown that the level of AM-PM is determined by the nonlinearity of the coupling transconductance. In any physical circuit, the coupling circuit will also contribute to the noise increase through its internal noise sources, which also increase with the coupling strength. This, together with the AM-PM noise addition considered here, then explains why the $3 \mathrm{~dB}$ noise reduction is rarely seen in measurements of this particular circuit.

\section{REFERENCES}

[1] A. Rofougaran, J. Rael, M. Rofougaran, and A. Abidi, "A 900MHz CMOS LC-oscillator with quadrature outputs," ISSC Tech. Dig. Papers, pp. 392-393, 1996.

[2] D. Cordeau, J.-M. Paillot, H. Cam, G. De Astis, and L. Dascalescu, "A fully monolithic SiGe quadrature voltage controlled oscillator design for GSM/DCS-PCS applications," 2002 IEEE RFIC Symp., pp. $455-458$.

[3] R. Aparicio and A. Hajimiri, "A noise-shifting differential Colpitts VCO," IEEE J. Solid State Circuits, vol. 37, no. 12, pp. 1728-1736, December 2002.

[4] P. Vanassche, G. Gielen, and W. Sansen, "Behavioral modeling of (coupled) harmonic oscillators," IEEE Trans. Computer-Aided Design, vol. 22, no. 8, pp. 1017-1026, August 2003.

[5] P. Andreani, A. Bonfanti, L. Romano, and C. Samori, "Analysis and design of a 1.8-GHz CMOS LC quadrature VCO," IEEE J. Solid State Circuits, vol. 37, no. 12, pp. 1737-1747, December 2002.

[6] L. Romano, S. Levantino, A. Bonfanti, C. Samori, and A. L. Lacaita, "Phase noise and accuracy in quadrature oscillators," vol. 1. ISCAS 2004, 2004, pp. 161-164.

[7] J. van der Tang, P. van de Ven, D. Kasperkovitz, and A. van Roermund, "Analysis and design of an optimally coupled 5-GHz quadrature LC oscillator," IEEE J. Solid State Circuits, vol. 37, no. 5, pp. 657-661, May 2002.

[8] T. Djurhuus, V. Krozer, J. V. r, and T. K. Johansen, "Am to pm noise conversion in a cross-coupled quadrature harmonic oscillator," IEEE Trans. Circuit and Syst. II, submitted.

[9] C. Samori, A. Lacaita, F. Villa, and F. Zappa, "Spectrum folding and phase noise in LC tuned oscillators," IEEE Trans. Circuit and Syst. II, vol. 45, no. 7, pp. 781-790, July 1998.

[10] D. Leeson, "A simple model of feedback oscillator noise," Proc. IEEE, vol. 54, pp. 429-430, February 1966. 